\title{
MARIA THERESIA UND DIE REFORM
}

\author{
KÁROLY KÓKAI \\ Universität Wien, Abteilung für Finno-Ugristik \\ karoly.kokai@univie.ac.at
}

\begin{abstract}
Maria Theresia gilt als Reformerin. Unbestritten hat sie den Reformbedarf ihrer Zeit wahrgenommen. Die anstehenden Reformen betrafen die Bauernbefreiung, die Einführung der Schulpflicht, die Einrichtung einer modernen Gesundheitsvorsorge, die religiöse Toleranz, ein progressives Justizsystem sowie die Förderung eines zukunftsweisenden kulturellen Aufschwungs. Der Beitrag listet die einzelnen Maria Theresia zugeschriebenen Reformen auf und versucht zu bestimmen, was in den konkreten Fällen „Reform“ heißt. Insbesondere wird der Frage nachgegangen, wofür Reform einerseits in der ungarischen Geschichtsschreibung und andererseits in der Epoche der Aufklärung steht, und inwiefern die Reformen von Maria Theresia diesen entsprechen.
\end{abstract}

Schlüsselwörter: Reform, Maria Theresia, Aufklärung, Barock, Ungarn

Was ist eine Reform? Das Wort ist zusammengesetzt aus dem lateinischen re: zurück und formare: bilden bzw. gestalten, heißt also: Wiederherstellung. Was wiederhergestellt werden soll, ist eine vergangene Ordnung, d.h. ein System, das funktioniert hat und auf das die Zukunft gebaut werden kann. Reform verspricht einen Fortschritt und zwar einen planvollen und kontinuierlichen. ${ }^{1}$ Reform in ihrem gegenwärtigen Sinn bezeichnet also zielgerichtete Veränderungen, die entscheidend, also tiefwirkend und nachhaltig sind.

Reform gab in Ungarn zwei Epochen den Namen: der Reformation und der Reformkor, was ins Deutsche übersetzt Reformzeit heißt. Die erste hat eine europäische Dimension, die zweite ist ein spezifisch ungarisches Phänomen. Was hat das nun mit Maria Theresia, also mit der Zeit von 1717-1780 zu tun?

Maria Theresia war bekanntlich eine tief katholische Person, die die katholische Religion als habsburgische Staatsideologie ansah. Sie lebte in der letzten Phase der Gegenreformation bzw. wenn man die mit dieser verbundene kulturhistorische Epoche nimmt, in der letzten Phase des Barock. Was Maria Theresia also mit Reformation zu tun hat, ist einfach zu sagen: Sie war deren personifiziertes Gegenteil.

In der österreichischen Geschichtsschreibung redet man bezüglich des 18. Jahrhunderts über drei Phasen von Reformen, die auch für drei verschiedene Zugänge zu dieser stehen sollen, nämlich von den theresianischen, josephinischen und leo- 
poldinischen. Die erste davon soll für die milde, die zweite für die überhitzte und die dritte für die abgebrochene Version stehen. Die Reformen von Maria Theresia werden so dem übertriebenen Reformeifer ihres ältesten Sohnes Joseph sowie den durch den frühen Tod nicht verwirklichten Reformen ihres jüngeren Sohnes Leopold gegenübergestellt. Letzterer konnte seine Vorstellungen als Großherzog der Toskana zwar bis 1790 verwirklichen, als Kaiser und König allerdings während der beiden Jahre von 1790 bis 1792 nicht umfassend umsetzen. Die zwei Jahre reichten gerade aus, die unter Joseph aufgestauten Konflikte - so in Ungarn - abzumildern. Was darauf folgte, war die Herrschaft von Franz II. von 1792 bis 1835, also jene Periode, an deren Ende in der ungarischen Geschichte die so genannte Reformzeit steht. Diese Reformzeit fängt mit der Publikation der Ergebnisse der Arbeit von parlamentarischen Kommissionen mit einer breiten öffentlichen ${ }^{2}$ Diskussion an, die um 1830 einsetzte - was laut ungarischer Geschichtsschreibung die Modernisierung des Landes einleitete. In dieser Interpretation konnte also erst um 1830, nach langen Jahren, Jahrzehnten und Jahrhunderten der verhinderten Entfaltung, was in unserem Zusammenhang heißt: 50 Jahre nach dem Tod von Maria Theresia, die Zeit der echten Reformen anbrechen. ${ }^{3}$

Beide Epochen, sowohl die Reformation als auch die Reformzeit, stehen also in der Geschichte Ungarns für etwas, was dem widerspricht, wofür die katholische Herrscherin, respektive die österreichische Reformerin stehen soll. Angesichts der hier erscheinenden verschiedenen Epochenbezeichnungen und Geschichtsinterpretationen stellt sich daher die Frage: Was meinen wir mit Reform, wenn wir über Maria Theresia sprechen?

Was Maria Theresia war und wofür sie steht, ist eine komplexe Angelegenheit, auch im Vergleich mit den restlichen Habsburgern. So steht sie als Herrscherin, und zwar als „Kaiserin“, im Vordergrund, obwohl nicht sie, sondern ihr Vater, ihr Mann und ihr Sohn Kaiser waren. ${ }^{4}$ Da für die Historie die Kontinuität des Hauses Habsburg das Entscheidende ist, einigte man sich jedoch auf die Sprachregelung, die bis heute gilt. Auch weil sie als einzige Frau in einer langen Reihe von Männern als Repräsentanten von Österreich dasteht, wurde ihr dieser Sonderstatus eingeräumt.

Unsere Konstruktion von Maria Theresia enthält also einige Klischees. Diese Klischees lassen sich in zwei Gruppen einteilen. Zur ersten gehört die gütige Landesmutter, die junge Königin, die von außen bedroht (in den österreichischen Erbfolgekriegen mit Preußen und Frankreich) ihr Reich rettet, die Herrscherin, die sich dem Wohl ihres Reiches opfert (Staatsgeschäfte einerseits und Familie andererseits) und die treue Witwe, die Ruhe und Stabilität signalisiert (im Gegensatz zum sie betrügenden Ehemann und dem die dritte Heirat verweigernden ältesten Sohn). Zur zweiten Klischee-Gruppe gehört die intolerante Herrscherin, die österreichische Protestanten in die ungarische Verbannung schickte, die erzkonservative Moralistin, die Sittenkommissionen einsetzte, die Antiaufklärerin, 
die die Folter verordnete und die Zensur verstärkte. Es gibt also positive und negative Klischees, die sich in Biographien und Ausstellungen, in Krisenzeiten und in Jubiläumsveranstaltungen (so 2017: dreihundert Jahre Geburt, 2030: zweihundertfünfzig Jahre Tod, 2040: dreihundert Jahre Herrschaftsantritt usw. usf.) hervorholen und aufwärmen lassen. In Österreich gilt Maria Theresia heute als positiv konnotierte historische Figur, als eine Frau, die dem Land Ansehen, Ruhe und Ordnung brachte. ${ }^{5}$ Dies befriedigt den Bedarf unserer Zeit nach einer feministisch korrekten Person, und lässt sich zugleich auch bewerkstelligen, ohne der Geschichte allzu große Gewalt anzutun. Mit der Glorifizierung einer großen Vergangenheit und der Beschwörung von Frieden und Fortschritt haben wir zugleich genau das, was die Habsburg-Ideologie, und so insbesondere Maria Theresia, wollte. $\mathrm{Zu}$ diesem Bild gehört auch das Klischee der sanften Reformerin, die der sturen Fortschrittsverhinderung der Ungarn gegenübergestellt wird. ${ }^{6}$

Wer war Maria Theresia? Sie war ab 1740 das Oberhaupt der Familie Habsburg, ab 1741 Königin von Ungarn und Herrscherin der Länder der ungarischen Krone. Sie war also als Maria II. rex hungariae, also König von Ungarn und damit Königin von Kroatien und Slawonien sowie Fürstin von Siebenbürgen. Bei ihrer Krönung in Pressburg legte sie einen Eid auf das Diploma Inaugurale ab, mit dem sie die Gesetze Ungarns, somit auch jene bezüglich des Wirksamwerdens von Verordnungen, anerkannte. ${ }^{7}$ Die repräsentativen Darstellungen von Franz Messmer, Franz Xaver Messerschmidt und Franz Anton Maulbertsch halten diese Figur fest - ohne freilich auf die juristischen und politischen Details einzugehen, die mit der komplexen historischen Situation gegeben war. Damit haben wir also wiederum die symbolischen Darstellungen einerseits und die historischen Details andererseits, die beträchtlich auseinanderklaffen können.

Was sind die Reformen, über die wir hier sprechen? Ist es die Bauernbefreiung? Die Einführung der Schulpflicht? Die Einrichtung einer modernen Gesundheitsvorsorge? Die religiöse Toleranz? Ein progressives Justizsystem? Die Förderung eines zukunftsweisenden kulturellen Aufschwungs? Ja, genau diese. Das sind ja die Bereiche, an denen eine Herrscherin der Zeit der Aufklärung in der langfristigen Entwicklungsgeschichte der europäischen Zivilisation gemessen wird.

Ich werde mich im Folgenden den Fragen, wer Maria Theresia war und was „Reform“ hier meint, von vier Seiten nähern.

Man kann sich erstens diesen Fragen nähern, indem man sich die historischen Umstände vergegenwärtigt, die zu jenen Reformen führten. Diese historischen Umstände waren sowohl außenpolitisch als auch innenpolitisch bedingt. Es gab also den Bedarf, innenpolitisch eine stabile Lage zu schaffen, um den außenpolitischen Herausforderungen der Zeit gerecht werden zu können. Innen war das Reich ja gespalten - indem es aus einer Reihe von Ländern bestand, die unterschiedliche rechtliche Stellungen hatten, so die Erbländer einerseits und Ungarn andererseits, aber auch innerhalb der Erbländer, siehe Böhmen einerseits 
und etwa Nieder- und Oberösterreich andererseits, bzw. innerhalb der Länder der ungarischen Krone - und überholungsbedürftig - wenn nicht anders, aus dem Grund, dass die Zeit rasante Änderungen, etwa im Bereich des Militärwesens und der Wirtschaft, aber auch was die politische Ideengeschichte betraf, mit sich brachte -, von außen wurde es von den intensiven Entwicklungen der Aufklärung einerseits und von der imperialen Konkurrenz aus dem Reich der Bourbonen und der Hohenzoller bedroht. Wollte Maria Theresia ihre Herrschaft retten, musste sie diesen Gefahren begegnen.

Innerhalb der deutschen Länder war, auch für Maria Theresia selbst, der Vergleich des Habsburger Reichs mit Preußen das Naheliegendste. Preußen war zwar flächenmäßig kleiner als das Habsburger Reich, militärisch aber mächtiger. Was die Reformen betrifft, war ersteres ebenfalls fortschrittlicher und dynamischer als zweiteres. Diese Diskrepanz, die sich auch im militärischen und dem anschließenden fiskalen Erfolg Friedrichs II. in Schlesien - jener Provinz, die Österreich in einem Krieg weggenommen wurde und in der nach der Übernahme deutlich mehr Steuern erhoben werden konnten - zeigte, machte Maria Theresia auch klar, dass sie handeln musste. Dieses Handeln war in den Regionen, wo Maria Theresia ohne eigenständigen Landtag und ohne Zwang zur Rücksicht auf die traditionellen Gesetze, also als Erzherzogin von Österreich und als Königin von Böhmen herrschte, leichter durchführbar als in den Regionen, wo es einen Landtag und eine Gesetzeskontinuität gab, also, wo sie als Königin von Ungarn herrschte. Hier galten nämlich ihre Verordnungen erst, wenn sie vom Königlichen Statthaltereirat verkündet und das heißt, von den Ungarn als gesetzeskonform akzeptiert wurden. Soweit die erste, den historischen Kontext betreffende Annäherung.

Ihre Reformen betrafen zweitens Bereiche wie Administration, Steuer, Zoll, Gesundheit, Unterricht, Kirche und Kultur. Da es hier um Maria Theresia als ungarische Königin geht, sollten wir fragen, was diese Reformen in Ungarn bedeuteten.

Ziel der Umgestaltung der Administration war es, eine effektive Verwaltung einzurichten. Der Weg dazu war die Errichtung von neuen Strukturen und Ämtern und die Anstellung neuer Beamten, die also bereit waren, den aktuellen Vorgaben gemäß zu arbeiten. So gründete Maria Theresia 1742 auf der obersten Verwaltungsebene die Hof- und Staatskanzlei (später umbenannt in „Haus-, Hof- und Staatskanzlei“"), führte weiter darunter so genannte Kreisämter ein, die mithin auf lokaler Ebene die Interessen des Staates vertraten und initiierte eine Steuerreform, um die Armee aufrüsten zu können. Das betraf aber eben die österreichischen und die böhmischen Länder, wo sie also als Königin von Böhmen und als Erzherzogin von Österreich herrschte. Ungarn betreffend, gab es nach wie vor die Cancellaria Regia Aulica Hungarica, die Ungarische Hofkanzlei in Wien, sowie das Consilium Regium Locumtenentiale Hungaricum, den Königli- 
chen Statthaltereirat und die Camera Regia Hungarica, die Ungarische Kammer, beide in Pressburg angesiedelt.

Die Conscriptio Animarum, wörtlich die „Seelenzusammenschreibung“, erfolgte in den 1760er- und 1770er-Jahren in gewissen Gebieten, so z.B. in der Diözese Veszprém in Ungarn alljährlich. Man könnte sie als Beginn der modernen Volkszählung ansehen. Wenn man die Sache näher betrachtet, zeigt sich allerdings, dass es hier nicht um eine Zählung der Gesamtbevölkerung, sondern um die Zusammenschreibung der katholischen Gläubigen handelte, und zwar aufgrund einer Verordnung des Papstes Paul V. aus 1614, was die apostolischen Visitationen vorbereiten sollte. Außerdem wurden nur Nichtadelige gezählt, da Adelige auf ihre Sonderrechte bestanden, in der tabellarischen Erfassung ihre persönlichen Rechte gefährdet sahen und daher nicht mitgezählt werden wollten.

Die Vectigal regni Hungariae partimque eidem annexarum, die Vectigal des Königreichs Ungarn und der dazu angehörigen Länder von 1754 war Teil der Regelung von zwei Zollgrenzen. Für die Außenzollgrenze des Reiches galten $20 \%$, für die Binnengrenze zwischen den Erbländern und Ungarn 5\% Maut. Ohne uns im Detail mit der Wirtschaftspolitik dieser Zeit auseinandersetzen zu müssen: Die Zollverordnung zeigt die Prinzipien der Wirtschaftspolitik des Reiches klar an: Es schottete sich nach außen mit einem relativ hohen Zoll ab und zog eine Binnengrenze auf.

1760 wurde die Praetoriana Nobilium Turma Hungarica, die Ungarische Adelige Leibgarde gegründet. Ihre Aufgabe war der diplomatische Kurierdienst und die Begleitung der Herrscherin. Nebenbei bezweckte man damit auch, loyale Ungarn zu erziehen.

Das sogenannte Urbarialpatent wurde 1767 erlassen. Dazu wurde eine Instructio pro commissariis regiis in re urbariali ad comitatus exmisis de serviens, also eine entsprechende Anleitung für die die Zusammenschreibung durchführenden Beamten herausgegeben. Ziel war, die Steuerpflichtigen, also die Bauern zu erfassen, um eine Grundlage für die Festlegung eines einheitlichen Steuersatzes zu schaffen. Die damit erzielte „Erleichterung“ für die Bauern war die Beschränkung der für den Grundherrn - also für den lokalen Adeligen - zu errichtende Robot, um die Staatseinnahmen optimieren zu können.

Die Constitutio Criminalis, das Strafgesetzbuch von 1769, war eine Aktualisierung der bestehenden Strafverordnung, und zugleich etwa mit dem Festschreiben der Folter auch ein Festhalten an voraufklärerischen juristischen Praktiken. Das Strafgesetzbuch galt zwar für die Erbländer und nicht für Ungarn, aber sehr wohl für ungarische Untertanen, wenn Sie auf dem Gebiet der Erbländer, also etwa in Wien, der Residenzstadt der ungarischen Königin, gefasst wurden; zweitens wurden Verbrecher, die diesem Gesetzbuch entsprechend behandelt wurden, wiederholt nach Ungarn abgeschoben. 
Das Generale Normativum in Re Sanitatis war ein Mandatum, also eine Beamtenverordnung. ${ }^{8}$ Sie ist die für Ungarn gültige lateinische Übersetzung des am 2. Januar 1770 erlassenen Hauptsanitätsnormativs, das entsprechend des Beschlusses des Ungarischen Statthaltereirats am 4. Oktober 1770 in Ungarn verkündet wurde. Es regelte erstens die Aufstellung von Ärzten, Chirurgen, Badern, Geburtshelferinnen und Apothekern und zweitens die Quarantäneverwaltung an den Grenzen.

Die Ratio educationis totiusque rei literariae per regnum Hungariae provincias eidem adnexas 1777 regulierte die Schulbildung. Heute würde man dafür „Lehrplan“" sagen. Sie galt für die katholischen Schulen und zwar auf allen drei Ebenen, also Volksschule, Gymnasium und Universität. Parallel dazu existierten natürlich auch protestantische Lehrpläne, auch solche aus der Zeit vor 1777. Die Übersiedlung der Universität von Tyrnau nach Ofen erfolgte 1777-1780. Das zu dieser Zeit erlassene Diploma Inaugurale gilt seither als ihre zweite Gründungsurkunde. Aus einer erzbischöflichen wurde nun eine königliche ungarische Institution. Eröffnet wurde sie im letzten Regierungsjahr von Maria Theresia, 1780.

Diese die Administration, die Steuer, die Leibgarde, das Strafgesetzbuch, das Gesundheitswesen und die Bildung betreffenden Verordnungen bilden also ein heterogenes Korpus, das zwar Ungarn berührte, aber von der Königin kaum unter Berücksichtigung der ungarischen Verhältnisse formuliert wurde.

Es ist, drittens, entscheidend, all das als Einzelelemente einer umfassenden Territorialpolitik anzusehen. Betrachtet man die Liste der Titel Maria Theresias, zeigt sich, dass sie mit der ungarischen Krone eine Reihe dieser Titel erbte. So war sie Königin von Kroatien, Dalmatien und Slawonien ${ }^{9}$ sowie Fürstin bzw. ${ }^{10}$ Großfürstin von Siebenbürgen. ${ }^{11}$ Die Territorialpolitik von Maria Theresia bestand aus dem Versuch, diese Tatsache zu nützen: Sie strebte an, die territoriale Integrität des Landes zu lockern bzw. weigerte sich, seine alte, d.h. bis ca. 1526/1527, bis zum Thronantritt von Ferdinand ${ }^{12}$ existierende, territoriale Integrität wiederherzustellen. Dies wurde verschiedentlich begründet, so als Reaktion auf das Zögern von Ungarn sich enger ins Habsburgerreich einzugliedern oder als militärische Notwendigkeit. Die südliche Grenze von Ungarn zum Osmanischen Reich, die sogenannte Militärgrenze bestand aus Teilen der ab 1718 wieder an Ungarn angegliederten Banschaft von Temeschwar, Banat genannt, weiters von Kroatien-Dalmatien-Slawonien, wobei Siebenbürgen ebenfalls sukzessive dieser Zone angegliedert wurde. Maria Theresia strebte also als ungarische Königin an, eine Südungarn und Ostungarn umfassende Sonderregion, die eben als Nicht-Teil von Ungarn zu gelten hatte, schrittweise weiter herauszubilden.

Der zweite wesentliche Punkt neben dem administrativen „Sonderstellen“ ist die Ansiedlungspolitik. Diese war durch dasselbe Interesse geleitet, die Integrität des Landes zu schwächen und zwar diesmal durch die Verstärkung des nicht-ungarischen Bevölkerungsanteils. Inpopulations- und Ansiedlungsprogramme gab 
es in Südungarn (durch Deportationen von österreichischen Protestanten oder von Kriminellen mittels jährlich zweimal stattfindender Abschiebungsaktionen, „Temeschwarer Wasserschub“ genannt, sowie durch Anwerbung von Siedlern), in Westungarn (durch Unterstützung des spontanen Zuzugs von Nicht-Ungarischsprachigen, nämlich Deutschen und Kroaten) und in Nordungarn (im Zuge der Industrialisierung im Bereich Bergbau und Metallverarbeitung mit böhmischen und deutschen Facharbeitern). Sowohl die administrativen Maßnahmen zwecks einer Sonderstellung von Südungarn und Ostungarn als auch die Ansiedlungsaktionen im Westen, Norden und Süden waren Prozesse, die nicht von Maria Theresia initiiert wurden. Sie setzte eine seit den osmanischen Kriegen existierende, auf Ungarn bezogene Politik fort. Bezüglich Maßnahmen innerhalb dieser Politik wäre also der Begriff Reform verfehlt, da dieser eine Initiative von systematischen Neuerungen bedeutet; was aber stattfand, war die Adaptierung der bis dahin praktizierten Politik für sich ergebende Möglichkeiten.

Es wäre viertens zu fragen, wofür Maria Theresia und ihre Reformen innerhalb der großen geistigen Entwicklung der Zeit, der Aufklärung, stehen. Betrachtet man die langfristigen Entwicklungen, dann ist die Aufklärung eine geistige Bewegung, die etwas fortsetzt, was mit dem Humanismus bereits vor dem Besetzen des ungarischen Throns durch die Habsburger aufbrach, Teile des 17., 18. und 19. Jahrhunderts dominierte und zu etwas führte, das unsere heutige Welt bestimmt. Aufklärung ist also keineswegs etwas, das 1741 plötzlich da war und für alle und sofort eine eindeutige Form annahm. Vielmehr ist Aufklärung und die mit ihr einhergehende Modernisierung ein widersprüchlicher, krisenhafter, in verschiedenen regionalen Versionen erscheinender, in unterschiedlichen Bereichen (Wissenschaft, Kultur, Politik, Gesellschaft, Wirtschaft) mit Phasenverschiebungen auftretender Prozess. Was hieß dies zur Zeit, als Maria Theresia ungarische Königin war?

Aufklärungsproblematische Verordnungen Maria Theresias, etwa die religiöse Intoleranz oder die Folter und die Hexenverfolgung betreffend, zeigen es klar an: Wenn Aufklärung eine bestimmte Geistigkeit meint, etwas, das durch ein naturwissenschaftliches Weltbild geprägt wurde, das Demokratisierungsprozesse förderte und Menschenrechte in den Vordergrund stellte, für Säkularisation, Fortschritt und Moderne stand, dann ist Maria Theresia nicht diejenige, die diese Geistigkeit vertrat, sondern im Gegenteil, die ihr klar entgegenstand. Dass in der Zeit ihrer Herrschaft Reformen passierten, kann also nicht diesem aufklärerischen Geist entsprungen sein. Klar ist zugleich, dass der Geist der Aufklärung zu ihrer Herrschaftszeit Ungarn erreichte und zwar nicht zuletzt in Institutionen, die damals in Wien gegründet wurden.

Die Einbindung von Ungarn in die Politik des Reichs verlief auf verschiedenen Ebenen. So etwa auf den höheren: In die Ungarische Hofkanzlei, in den Königlichen Statthaltereirat und in die Ungarische Kammer wurden ja möglichst 
loyale Untertanen ernannt. Dasselbe geschah aber auch weiter unten. Im 1746 gegründeten Theresianum, einer Art Verwaltungsakademie studierten in den ersten drei Jahrzehnten seines Bestehens ca. einhundert Ungarn, so z.B. ab 1772 Ferenc Széchenyi, der wenige Jahrzehnte später das ungarische Nationalmuseum und die ungarische Nationalbibliothek initiierte.

Die ungarische adelige Leibgarde versah ab 1760 repräsentative Aufgaben. Die Leibgardisten wurden auch ausgebildet, so studierten sie neben Militärwesen auch Sprachen und zwar Deutsch und Französisch (sowie weitere fakultative Fächer). Stationiert waren sie in Wien, am Anfang ca. 100 junge Männer. Bekanntlich übersetzten die Gardisten in ihrer Freizeit französische und deutsche Texte ins Ungarische. Sie mussten 4 Jahre dienen. Im Falle von György Bessenyei, des geistesgeschichtlich Einflussreichsten, waren es zweimal vier Jahre zwischen 1765 und 1773. Während dieser Dienstzeit, 1772, publizierte er Ágis tragédiá$j a$, ein Drama, das den Generationskonflikt zwischen konservativen Vätern und fortschrittlichen Söhnen thematisiert. Er blieb nach Ende seines Dienstes auch in Wien und arbeitete an seinem literarischen Werk weiter. 1779 erschien eine Sammlung der so entstandenen Texte mit dem Titel A Holmi. Sie enthält philosophische Abhandlungen, Essays und Dialoge. Was hier passierte, war der Anfang der Aufklärung in Ungarn, und somit das Gegenteil dessen, was vorgesehen war. Es wurde nicht eine dem Thron von Habsburg gegenüber loyale Jugend erzogen, die dann den habsburgischen Machtinteressen gemäß die ungarische Politik mit umgestaltete, sondern eine Generation, die eine den habsburgischen Traditionen und den habsburgischen Interessen widersprechende echte Erneuerungsbewegung einleitete.

Ein wichtiger Teil der modernen Öffentlichkeit, der Zivilgesellschaft, ist die Presse. Zwei Meilensteine in diesem Bereich waren 1764 das Erscheinen der Pressburger Zeitung und 1780 von Magyar Hirmondó, beide in Pressburg, im politischen Zentrum von Ungarn. Hier handelte es sich zunächst ebenfalls um einen kleinen Kreis, also mit heutigen Kategorien gemessen, eher um eine geschlossene Gesellschaft als um eine echte Öffentlichkeit - die jedoch wiederum den Keim einer Zukunft enthielt.

Nimmt man den Bereich Kultur, wird offenbar, was hier Reform heißt. Zur Zeit der Herrschaft von Maria Theresia startete in ihrer Residenzstadt Wien die neuere ungarische Literatur, in der Krönungsstadt Pressburg, die auch die wichtigsten ungarischen Ämter beherbergte, erschienen die ersten zuerst deutschsprachigen und dann ungarischen Zeitschriften. Das war mehr als Reform, nämlich ein echter Beginn, ein Wendepunkt, ein epochaler Aufbruch. Dies alles geht aber keinesfalls auf Initiativen von Maria Theresia oder ihrer Berater zurück. Ganz im Gegenteil. Es ist aus ihrer Perspektive betrachtet im besten Fall ein ungewolltes Nebenprodukt. Und es zeigt an, dass bei Maria Theresia über „Reform“ zu sprechen irreführend ist, obwohl was während ihrer Herrschaft passierte genuin das 
ist, was Reform im eigentlichen Sinne meint: den Beginn einer einschneidenden, tiefwirkenden und nachhaltigen Entwicklung.

Was wir somit haben ist, wenn man das so nennen will, eine paradoxe Situation. Die Reformen Maria Theresias waren keine Reformen, sondern Versuche, die Effektivität des Konservativismus zu erhöhen und die Reformen, die diesen Namen verdienen - siehe z.B. die Reformation und die Gegenreformation - zu verhindern, wenn nicht rückgängig zu machen.

Was sind also die Reformen, über die hier gesprochen werden kann? Die Bauernbefreiung fand in Ungarn 1848 statt. Die Einführung der Schulpflicht erfolgte in Ungarn unter dem Kultusminister Josef Eötvös nach dem Ausgleich 1867. Die Einrichtung einer modernen Gesundheitsvorsorge war erst im Laufe des 20. Jahrhunderts möglich. Die religiöse Toleranz ist eine Errungenschaft der Säkularisation, und wie die gegenwärtigen europäischen Aggressionen gegen Andersgläubige zeigen, wurde sie bis heute nicht verwirklicht. Ein progressives Justizsystem entwickelte sich erst langsam, wobei einige diesbezügliche Probleme bis heute ungelöst sind..$^{13}$ Die Förderung eines zukunftsweisenden kulturellen Aufschwungs kann ihr ebenfalls nicht zugeschrieben werden. Das geschah ja mit dem Erscheinen von ungarischsprachigen literarischen Werken und Zeitschriften, zwar in den habsburgischen Machtzentren Wien und Pressburg, aber in einer Sprache, die die Königin von Ungarn nicht beherrschte.

Können wir hier also über Reformen sprechen, oder gehört das alles eher zur barocken Vielfalt der Hofbürokratie mit Relazionen, Edikten, Reskripten, Dekreten, Handschreiben, Mandaten, Patenten und Diplomen? Wir sollten eben über Ratio Educationis, Conscriptio Animorum, Robotpatent, Gründungsdiplom der Praetoriana Nobilium Turma Hungarica, über Constitutio Criminalis, über Verordnungen, den Temeschwarer Wasserschub und das Ansiedlungs- und Inpopulationswesen betreffend, sprechen. Das Wort Reform ist nämlich zur Zeit von Maria Theresia niemandem, am wenigstens ihr, in den Sinn gekommen. Heute - und das ist der Punkt, den ich mit diesem Aufsatz über Maria Theresia und die Reform hervorheben möchte - wäre es daher wissenschaftlich unhaltbar, diesbezüglich den Begriff „Reform“ einzusetzen. Ihr „Reformwille“ machte spätestens an der Grenze von Ungarn Halt, und zwar deshalb, weil sie nie gewillt war, sich mit der organischen Entwicklung des Landes auseinanderzusetzen.

\section{Anmerkungen}

1 Reform wird im politischen Sinne oft der Revolution entgegengesetzt, was die gewaltlose Seite der einen und das Abrupte, Ruckartige der zweiten betont. In der ungarischen Geschichtsschreibung unserer Zeit ist der Begriff Revolution außerdem durch die langen Jahrzehnte der marxistischen, respektive kommunistischen Geschichtsideologie immer noch stark besetzt und meint immer noch Schritte im Befreiungskampf des Proletariats. 
2 Wobei natürlich sowohl „breit“ als auch „öffentlich“ relative Begriffe sind und hier im Sinne von Ungarn um 1830 zu verstehen sind. Gemäß heutigen Kategorien fanden diese Diskussionen in engen und geschlossenen Kreisen der damaligen politischen Elite statt und dort auch innerhalb einer - nämlich in der fortschrittlichen - Fraktion.

3 Den Beginn markieren der Landtag 1825-1827, die Publikation der Ergebnisse der Arbeit der 1792 eingesetzten Commissionen, und die Publikation von István Széchenyis Credit 1830.

4 Römisch-Deutsche Kaiser waren Karl VI. (in Ungarn III. Károly) 1711-1740, Franz I. Lothringen 1745-1765 und Joseph II. Habsburg-Lothringen 1765-1790.

5 - obwohl sie ca. während der Hälfte ihrer Regierungszeit Kriege führte: Österreichischer Erbfolgekrieg 1740-1748, siebenjähriger Krieg 1756-1763, Bayerischer Erbfolgekrieg 17771779.

6 siehe beispielsweise Pieter Judson: The Habsburg Empire 2016.

7 Am 24. Juni 1741 unterschrieb sie das Diploma Inaugurale (Antrittsdiplom), formuliert durch den Hofkanzler Ludwig Batthyány. Gekrönt wurde sie am 25. Juni in der St. Martin Kirche. Anschließend legte sie den Eid auf das Diplom ab.

8 Es war kein Gesetz, da nach 1765 keine Landtagsitzungen mehr stattfanden, wo eine Verordnung Gesetzeskraft hätte erlangen können.

9 Die drei Länder wurden ab 1744 als ein eigenes Königreich angesehen.

$10 \mathrm{Ab} 1768$.

11 Der Titel lautete „Princeps Transsylvaniae partiumque regni Hungariae dominus“ d.h. Fürst von Siebenbürgen und Herr der Teile des Ungarischen Königreichs.

12 Ferdinand I. Habsburg wurde am 17. Dezember 1526 zum ungarischen König gewählt und am 3. November 1527 gekrönt.

13 So weiß die Gesellschaft bis heute nicht mit ihren Verbrechern etwas anzufangen. Wegsperren, Rache in der Form von abschreckenden Strafen, Demütigung durch Polizei- und Justizvollzugsbeamte, das Heranzüchten von Kriminellen während langer Gefängnisaufenthalte, Stigmatisierung sind nach wie vor die hier praktizierten Methoden. 